\title{
ON LINKING DOUBLE LINES
}

\author{
BY
}

JUAN MIGLIORE

\begin{abstract}
A double line is a nonreduced locally Cohen-Macaulay scheme of degree two supported on a line in projective three-space. The heart of this work is to compute the associated Hartshorne-Rao module for such a curve. We can then say exactly when two such curves are in the same liaison class and in fact when they are directly linked. In particular, we find that $C$ is only self-linked in characteristic two.
\end{abstract}

Introduction. Let $k$ be an algebraically closed field and $S=k\left[X_{0}, X_{1}, X_{2}, X_{3}\right]$. A double line $C \subset \mathbf{P}_{k}^{3}$ is a nonreduced locally Cohen-Macaulay scheme of degree two supported on a line. The main purpose of this paper is to determine when two such curves can be linked. This is accomplished by a careful study of the Hartshorne-Rao module $M(C)=\bigoplus_{n \in \mathbf{Z}} H^{1}\left(\mathbf{P}^{3}, I_{C}(n)\right)$ (cf. [R1]) and has a somewhat surprising answer. In addition, we check when $C$ can be self-linked.

In order to state the results, we first recall briefly the description of double lines due to Harris (cf. [H, pp. 32-33] for more detail). He gives a geometric description of a double line $C$ in $\mathbf{P}^{3}$, produces the homogeneous ideal $I(C)$, then verifies that the degree is in fact two and calculates the arithmetic genus.

To specify a double line $C$ in $\mathbf{P}^{3}$ one has to choose the underlying line $\lambda$ and then specify for each point $P \in \lambda$ a normal direction to $\lambda$. This is done by specifying for each $P \in \lambda$ a plane $H_{P}$ containing $\lambda$. Equivalently, we have a map $\psi: \lambda \rightarrow \mathbf{P}^{3} / \lambda$ (where $\mathbf{P}^{3} / \lambda \cong \mathbf{P}^{1}$ represents the pencil of planes through $\lambda$ ).

Without loss of generality, let $\lambda$ be the line given by $X_{2}=X_{3}=0$. Then we can write

$$
\psi\left(\left[a_{0}, a_{1}, 0,0\right]\right)=F\left(a_{0}, a_{1}\right) X_{2}+G\left(a_{0}, a_{1}\right) X_{3},
$$

where $F$ and $G$ are homogeneous polynomials of degree equal to $\operatorname{deg} \psi=d$ (say), with no common zero. We can also think of this as a map of $\mathbf{P}^{1}$ to $\mathbf{P}^{1}$ :

$$
\psi\left(\left[X_{0}, X_{1}\right]\right)=\left[F\left(X_{0}, X_{1}\right), G\left(X_{0}, X_{1}\right)\right] .
$$

This information is lumped together in the ideal of $C$, which is

$$
I(C)=\left(X_{2}^{2}, X_{2} X_{3}, X_{3}^{2}, X_{2} F\left(X_{0}, X_{1}\right)-X_{3} G\left(X_{0}, X_{1}\right)\right) .
$$

Note that $X_{2} F-X_{3} G$ corresponds to a surface $S$ of degree $d+1$ which is nonsingular in a neighborhood of $\lambda$ (since $F$ and $G$ have no common zero). So we can equivalently write

$$
I(C)=\left(I(\lambda)^{2}, I(S)\right) .
$$

Finally, Harris gives an elementary argument to show that the Hilbert polynomial for $C$ is $2 x+d+1$. Hence the degree of $C$ is 2 and the arithmetic genus $p_{a}(C)=-d$.

Received by the editors September 24, 1984.

1980 Mathematics Subject Classification. Primary 14H99; Secondary 13C99. 
We can also compute the Hilbert function for $C$. For $n \leq 1$ this is trivial. For $n \geq 2$ observe that any element of $I(C)$ of degree $n$ can be uniquely expressed in the form

$$
X_{2}^{2} P\left(X_{0}, X_{1}, X_{2}\right)+X_{2} X_{3} Q\left(X_{0}, X_{1}, X_{2}, X_{3}\right)+X_{3}^{2} R\left(X_{0}, X_{1}, X_{3}\right) \quad(n \leq d)
$$

or

$$
\begin{aligned}
X_{2}^{2} P\left(X_{0}, X_{1}, X_{2}\right) & +X_{2} X_{3} Q\left(X_{0}, X_{1}, X_{2}, X_{3}\right)+X_{3}^{2} R\left(X_{0}, X_{1}, X_{3}\right) \\
& +\left[X_{2} F\left(X_{0}, X_{1}\right)-X_{3} G\left(X_{0}, X_{1}\right)\right] S\left(X_{0}, X_{1}\right) \quad(n \geq d+1),
\end{aligned}
$$

where $P, Q$ and $R$ are homogeneous of degree $n-2$ and $S$ is homogeneous of degree $n-d-1$. Therefore

$$
\operatorname{dim}\left(\frac{S}{I(C)}\right)_{n}= \begin{cases}\left(\begin{array}{c}
n+3 \\
3
\end{array}\right)-\left(\begin{array}{c}
n \\
2
\end{array}\right)-\left(\begin{array}{c}
n+1 \\
3
\end{array}\right)-\left(\begin{array}{c}
n \\
2
\end{array}\right) & \text { if } n \leq d \\
\left(\begin{array}{c}
n+3 \\
3
\end{array}\right)-\left(\begin{array}{c}
n \\
2
\end{array}\right)-\left(\begin{array}{c}
n+1 \\
3
\end{array}\right)-\left(\begin{array}{c}
n \\
2
\end{array}\right)-(n-d) & \text { if } n \geq d\end{cases}
$$

Turning to liaison, it turns out that the cases $d=0$ and $d=1$ are trivial. The main result of the paper is the following:

THEOREM. Let $C$ and $C^{\prime}$ be double lines and assume $p_{a}(C) \leq-2$. Then $C$ is linked to $C^{\prime}$ if and only if

(a) they have the same line $\lambda$ as support,

(b) they have the same arithmetic genus $-d$,

(c) the corresponding maps $\psi, \psi^{\prime}: \lambda \rightarrow \mathbf{P}^{3} / \lambda$ differ by an automorphism of the target $\mathbf{P}^{3} / \lambda$.

To achieve this, $\S 1$ gives some preliminary results and $\S 2$ describes a special type of complete intersection which is used to simplify the computations. The heart of the paper is in $\S 3$ where we describe the global sections of the sheaves $0_{C}(n)$, compute the Hartshorne-Rao module $M(C)$, and prove the main theorem. $\S 4$ gives an interesting interpretation which is purely geometric, from which we establish the fact that double lines of arithmetic genus $\leq-2$ are only self-linked in characteristic two. (More generally, we see when $C$ and $C^{\prime}$ can be directly linked.)

ACKNOWLEDGEMENT. I am extremely grateful to J. Harris and to A. Landman for many very useful comments and suggestions.

1. First results. A key strategy for this paper will be to study the sheaves $O_{C}(n)$ for all $n$, which will tell us what we need to known about $M(C)$. In this section we give a first description of $O_{C}$, the main point being the exact sequence (3). As a consequence we compute the dimensions of the components of $M(C)$.

To begin, let $S$ be the surface corresponding to $X_{2} F-X_{3} G$ as in the Introduction. Then since $I(S) \subset I(C) \subset I(\lambda)$ it follows that $\lambda \subset C \subset S$.

LEMMA 1.1. On $S, \lambda \cdot \lambda=1-d$.

PROOF. This is an immediate application of the adjunction formula, recalling that $\omega_{S}=O_{S}(d-3)$ (since $\left.\operatorname{deg} S=d+1\right)$ so $K_{S}=(d-3) H$ (where $K_{S}$ is the canonical divisor class and $H$ is the class of a hyperplane section), and the geometric genus $\pi(\lambda)=0$ :

$$
0=\pi(\lambda)=\frac{\lambda \cdot \lambda+K_{S} \cdot \lambda}{2}+1=\frac{\lambda \cdot \lambda+(d-3)}{2}+1
$$


Now, we have an exact sequence of sheaves

$$
0 \rightarrow I_{C, S} \rightarrow I_{\lambda, S} \rightarrow I_{\lambda, C} \rightarrow 0
$$

from which we deduce

$$
I_{\lambda, S} / I_{C, S} \cong I_{\lambda, C}
$$

Since $\lambda \hookrightarrow C$ we have a natural map $O_{C} \rightarrow O_{\lambda}$, which yields the exact sequence

$$
0 \rightarrow I_{\lambda, C} \rightarrow O_{C} \rightarrow O_{\lambda} \rightarrow 0
$$

or, substituting the isomorphism (1) (thinking of this as a sequence of sheaves on S)

$$
0 \rightarrow I_{\lambda, S} / I_{C, S} \rightarrow O_{C} \rightarrow O_{\lambda} \rightarrow 0 \text {. }
$$

But since $I(C)=\left(I(\lambda)^{2}, I(S)\right)$ it follows that $I_{C, S}=I_{\lambda, S}^{2}$. Now, $I_{\lambda, S} / I_{\lambda, S}^{2}$ is the conormal bundle of $\lambda$ on $S$ (recall $S$ is smooth around $\lambda$ ), which is a line bundle of degree $-(\lambda \cdot \lambda)=d-1$ on $\lambda \cong \mathbf{P}^{1}$ (cf. [Ha, p. 361]). Therefore we can write (2) in the form

$$
0 \rightarrow O_{\lambda}(d-1) \stackrel{\alpha}{\rightarrow} O_{C} \stackrel{r}{\rightarrow} O_{\lambda} \rightarrow 0 .
$$

The maps $\alpha$ and $r$ will be described following Proposition 3.1.

From this we can quickly compute the dimensions of the components of the Hartshorne-Rao module $M(C)$ :

LEMMA 1.2 .

$$
\operatorname{dim} M_{n}(C)= \begin{cases}0, & n \leq-d \\ d+n, & -d \leq n \leq 0 \\ d-n, & 0 \leq n \leq d \\ 0, & n \geq d\end{cases}
$$

Proof. From the exact sequence of sheaves

$$
0 \rightarrow I_{C}(n) \rightarrow O_{P^{3}}(n) \rightarrow O_{C}(n) \rightarrow 0
$$

we get the associated long exact sequence in cohomology:

$$
0 \rightarrow H^{0}\left(\mathbf{P}^{3}, I_{C}(n)\right) \rightarrow H^{0}\left(\mathbf{P}^{3}, \mathcal{O}(n)\right) \rightarrow H^{0}\left(C, O_{C}(n)\right) \rightarrow M_{n}(C) \rightarrow 0 .
$$

Having (3) and twisting, we can compute $h^{0}\left(O_{C}(n)\right)$ for any $n$. (Note that

$$
h^{1}\left(O_{\lambda}(n+d-1)\right)=0 \text { for } n \geq-d \quad \text { and } \quad h^{0}\left(O_{\lambda}(n)\right)=0 \text { for } n \leq-1 \text {.) }
$$

Then recalling the Hilbert function calculation of the Introduction, the result is easily obtained using (4).

In other words, $\left\{\operatorname{dim} M_{n}(C) \mid n \in \mathbf{Z}\right\}$ is the sequence

$$
\ldots, 0,0,1,2, \ldots, d-1, d, d-1, \ldots, 2,1,0,0, \ldots
$$

where the "peak" occurs for $n=0$. The "diameter" of $M(C)$ is thus $2 d-1$, and 
this gives a necessary condition for liaison:

COROllary 1.3. If two double lines $C$ and $C^{\prime}$ are linked, then the corresponding maps $\psi: \lambda \rightarrow \mathbf{P}^{3} / \lambda$ and $\psi^{\prime}: \lambda^{\prime} \rightarrow \mathbf{P}^{3} / \lambda^{\prime}$ have the same degree $d$ (and, equivalently, $C$ and $C^{\prime}$ have the same arithmetic genus $-d$ ).

REMARK 1.4. It follows that a double line can have a Hartshorne-Rao module of arbitrarily large "diameter", that it can have arbitrarily large components, and that it can have arbitrarily many nonzero components in negative degrees. Furthermore, for $d>0, C$ is "extremal" in its liaison class in the following sense: Since $\operatorname{dim} M_{0}(C)>\operatorname{dim} M_{1}(C)$, it follows from Proposition 2.8 of $[\mathbf{M}]$ that no curve in the liaison class can have a Hartshorne-Rao module which is leftward shift of $M(C)$.

REMARK 1.5. A double line is also extremal in the sense that (for $d>0$ ) there is obviously no curve in the liaison class of strictly smaller degree. This paper describes which other degree two curves are in the liaison class.

For $d=0, C$ is a plane curve and hence a complete intersection. $M(C)=0$ and $C$ is arithmetically Cohen-Macaulay. $C$ is thus linked to any line and any (plane) conic in $\mathbf{P}^{3}$, including all other double lines with $d=0$.

For $d=1, M(C) \cong k$ so $C$ is arithmetically Buchsbaum (cf. [GMV2]). $C$ is linked to any pair of skew lines (cf. $[\mathbf{R} \mathbf{1}])$, as well as to any other double line with $d=1$.

For $d>1$, the components of $M(C)$ are as described above, but now there may be some nontrivial module structure. In fact, it was shown in [GMV2] that such a $C$ is never arithmetically Buchsbaum (i.e. there does exist nontrivial module structure). Because of this added complexity, it is reasonable to expect that not all double lines with the same $d$ will be linked.

2. Basic links. We are assuming that $C$ is a double line with ideal $\left(X_{2}^{2}, X_{2} X_{3}\right.$, $\left.X_{3}^{2}, X_{2} F\left(X_{0}, X_{1}\right)-X_{3} G\left(X_{0}, X_{1}\right)\right)$, where $\operatorname{deg} F=\operatorname{deg} G=d$. Observe that if $T$ is a reducible quadratic polynomial of the form $\left(\alpha X_{2}+\beta X_{3}\right)\left(\gamma X_{2}+\delta X_{3}\right)$ (i.e. the corresponding surface is the union of two planes, both containing the line $\lambda:\left(X_{2}=\right.$ $\left.X_{3}=0\right)$ ), then $T \in I(C)$.

If $T_{1}$ and $T_{2}$ are both of this form, with no common factor, then the corresponding complete intersection curve $X$ links $C$ to a curve $C^{\prime}$ which is again locally CohenMacaulay. Since $\operatorname{deg} X=4$ we have $\operatorname{deg} C^{\prime}=2$, and clearly $C^{\prime}$ is supported on $\lambda$. Then by Harris' description,

$$
I\left(C^{\prime}\right)=\left(X_{2}^{2}, X_{2} X_{3}, X_{3}^{2}, X_{2} F^{\prime}\left(X_{0}, X_{1}\right)-X_{3} G^{\prime}\left(X_{0}, X_{1}\right)\right),
$$

where $F^{\prime}$ and $G^{\prime}$ have no common root. Furthermore, by Corollary 1.3 we have $\operatorname{deg} F^{\prime}=\operatorname{deg} G^{\prime}=\operatorname{deg} F=\operatorname{deg} G=d$.

For our purposes it will actually be enough to look at complete intersections of the form $\left(T_{1}, T_{2}\right)$, where $T_{1}=\left(X_{2}\right)\left(X_{2}+\tau X_{3}\right)$ and $T_{2}=\left(X_{3}\right)\left(\mu X_{2}+X_{3}\right)$. Observe that $T_{1}$ and $T_{2}$ have no common factor (and hence give a link) if and only if the determinant $\left|\begin{array}{cc}1 & \mu \\ -\tau & -1\end{array}\right| \neq 0$. We shall call such a link a basic link.

Given $I(C)$ and the basic link $\left(T_{1}, T_{2}\right)$, we can compute the ideal of the residual double line $C^{\prime}$. Recall

$$
I\left(C^{\prime}\right)=\left(T_{1}, T_{2}\right): I(C)
$$


Since we know $I\left(C^{\prime}\right)=\left(X_{2}^{2}, X_{2} X_{3}, X_{3}^{2}, X_{2} F^{\prime}\left(X_{0}, X_{1}\right)-X_{3} G^{\prime}\left(X_{0}, X_{1}\right)\right)$ with $\operatorname{deg} F^{\prime}$ $=\operatorname{deg} G^{\prime}=d$, we have only to identify $F^{\prime}$ and $G^{\prime}$ in terms of $F, G, \tau$ and $\mu$.

By (5),

$$
\begin{aligned}
& {\left[X_{2} F^{\prime}\left(X_{0}, X_{1}\right)-X_{3} G^{\prime}\left(X_{0}, X_{1}\right)\right]\left[X_{2} F\left(X_{0}, X_{1}\right)-X_{3} G\left(X_{0}, X_{1}\right)\right]} \\
& \quad=P\left(X_{0}, X_{1}\right)\left(X_{2}\right)\left(X_{2}+\tau X_{3}\right)+Q\left(X_{0}, X_{1}\right)\left(X_{3}\right)\left(\mu X_{2}+X_{3}\right)
\end{aligned}
$$

for some $P, Q$. Then $F F^{\prime}=P$ and $G G^{\prime}=Q$, so one deduces that $F^{\prime}(-\tau F-G)=$ $G^{\prime}(\mu G+F)$. But $F^{\prime}$ and $G^{\prime}$ have no common root, nor do $(-\tau F-G)$ and $(\mu G+F)$. Therefore, up to scalar multiplication we have

$$
F^{\prime}=\mu G+F, \quad G^{\prime}=-\tau F-G .
$$

Equivalently,

$$
\left[\begin{array}{l}
F^{\prime}\left(X_{0}, X_{1}\right) \\
G^{\prime}\left(X_{0}, X_{1}\right)
\end{array}\right]=\alpha\left[\begin{array}{cc}
1 & \mu \\
-\tau & -1
\end{array}\right]\left[\begin{array}{l}
F\left(X_{0}, X_{1}\right) \\
G\left(X_{0}, X_{1}\right)
\end{array}\right]
$$

for some scalar $\alpha \neq 0$. We conclude

Proposition 2.1. Let $C$ and $C^{\prime}$ have ideals as above. Then $C$ is (directly) linked to $C^{\prime}$ by a basic link if and only if $\left[\begin{array}{c}F_{G^{\prime}}^{\prime} \\ ]\end{array}\right]=A\left[\begin{array}{l}F \\ G\end{array}\right]$, where $\operatorname{det} A \neq 0$ and $\operatorname{trace} A=$ 0 (but the main diagonal is not zero).

REMARK 2.2. We know that direct linking is a symmetric relation. Hence if we link $C$ to $C^{\prime}$ as above and then apply the same surfaces to $C^{\prime}$, we must get $C$ back. This is neatly reflected in the fact that

$$
\left[\begin{array}{cc}
1 & \mu \\
-\tau & -1
\end{array}\right]\left[\begin{array}{cc}
1 & \mu \\
-\tau & -1
\end{array}\right]=\left[\begin{array}{cc}
1-\tau \mu & 0 \\
0 & 1-\tau \mu
\end{array}\right]=(1-\tau \mu)\left[\begin{array}{ll}
1 & 0 \\
0 & 1
\end{array}\right]
$$

REMARK 2.3. Since

$$
\left[\begin{array}{cc}
1 & -1 \\
0 & -1
\end{array}\right]\left[\begin{array}{cc}
1 & 0 \\
2 & -1
\end{array}\right]\left[\begin{array}{cc}
1 & -1 \\
2 & -1
\end{array}\right]=\left[\begin{array}{ll}
1 & 0 \\
0 & 1
\end{array}\right]
$$

it follows that $C$ is linked to itself by a sequence of three basic links. (Note that by Hartshorne's theorem (cf. [R1]) this implies that $M(C)$ is self-dual.)

REMARK 2.4. As a corollary to Proposition 2.1, note that $C$ is self-linked (cf. [R2]) by a basic link if and only if char $k=2$ (and we take $\tau=\mu=0$ ). We shall say more about self-linkage in $\S 4$.

3. Main results. As indicated earlier, we are interested in studying the sheaf $\mathrm{O}_{C}(n)$. The key point is the following:

PROPOSITION 3.1. The global sections of $O_{C}(n)$ are of the form

$$
H^{0}\left(C, O_{C}(n)\right)=\left\{\begin{array}{l|l}
A\left(X_{0}, X_{1}\right)+B\left(X_{0}, X_{1}\right) \cdot \frac{X_{2}}{G\left(X_{0}, X_{1}\right)} & \begin{array}{l}
A \text { and } B \text { are polyno- } \\
\text { mials of degree } n \text { and } \\
d-1+n \text { respectively } \\
\text { or zero }
\end{array}
\end{array}\right\} .
$$

PROOF. We shall prove the inclusion $\supseteq$, and then the result will follow from a check of vector space dimensions, using sequence (3). 
Recall that $I(C)=\left(X_{2}^{2}, X_{2} X_{3}, X_{3}^{2}, X_{2} F\left(X_{0}, X_{1}\right)-X_{3} G\left(X_{0}, X_{1}\right)\right)$. Let $U_{0}$ be the open set $\left\{X_{0} \neq 0, G\left(X_{0}, X_{1}\right) \neq 0\right\}$ on $\mathbf{P}^{3}$, and consider the exact sequence

$$
0 \rightarrow \mathrm{O}_{C}(n)\left(U_{0}\right) \rightarrow \mathrm{O}_{\mathbf{P}^{3}}(n)\left(U_{0}\right) \rightarrow \mathrm{O}_{C}(n)\left(U_{0}\right) \rightarrow \cdots .
$$

For any open set $U, \boldsymbol{O}_{\mathbf{P}^{3}}(n)(U)=\{P / Q \mid \operatorname{deg} P=\operatorname{deg} Q+n, Q$ never zero on $U\}$. For $I_{C}(n)(U) \subset O_{\mathbf{P}^{3}}(n)(U)$ we have the further condition $P \in I(C \cap U)$.

Hence it follows that (up to isomorphism) at least the following inclusions must hold:

$$
\begin{aligned}
\bigcirc_{C}(n)\left(U_{0}\right) & \supseteq \frac{O_{\mathbf{P}^{3}}(n)\left(U_{0}\right)}{I_{C}(n)\left(U_{0}\right)} \\
& \supseteq\left\{\frac{A\left(X_{0}, X_{1}\right)}{X_{0}^{p}}+\frac{B\left(X_{0}, X_{1}\right) \cdot X_{2}}{X_{0}^{q} G\left(X_{0}, X_{1}\right)} \mid \begin{array}{l}
\operatorname{deg} A=p+n \\
\operatorname{deg} B=q+d-1+n
\end{array}\right\} .
\end{aligned}
$$

Similarly, on $U_{1}=\left\{X_{1} \neq 0, G\left(X_{0}, X_{1}\right) \neq 0\right\}$ we have

$$
\begin{aligned}
\mathcal{O}_{C}(n)\left(U_{1}\right) & \supseteq \mathcal{O}_{\mathbf{P}^{3}}(n)\left(U_{1}\right) / I_{C}(n)\left(U_{1}\right) \\
& \supseteq\left\{\frac{D\left(X_{0}, X_{1}\right)}{X_{1}^{s}}+\frac{E\left(X_{0}, X_{1}\right) \cdot X_{2}}{X_{1}^{t} G\left(X_{0}, X_{1}\right)} \mid \begin{array}{l}
\operatorname{deg} D=s+n \\
\operatorname{deg} E=t+d-1+n
\end{array}\right\} .
\end{aligned}
$$

Two such sections patch together to give a section over $U=U_{0} \cup U_{1}$ if and only if $p=q=s=t=0$, as in the statement of the proposition. But such a section over $U$ in fact extends to a global section of $O_{C}(n)$ (i.e. it extends to $\left\{G\left(X_{0}, X_{1}\right)=0\right\}$ ) because on $C$ we have $X_{2} / G=X_{3} / F$ and $F$ and $G$ have no common root.

REMARK 3.2. As a result of this proposition, we may view the map $\alpha$ in the exact sequence (3) as multiplication by $X_{2} / G\left(X_{0}, X_{1}\right)$ and $r$ as the restriction to the first term.

From Proposition 3.1 we can immediately describe $M(C)$ :

COROLlaRY 3.3. $M(C) \cong\left[k\left[X_{0}, X_{1}\right] /(F, G)\right](d-1)$ as an $S$-module.

ProOF. We have the usual exact sequence (4):

$$
0 \rightarrow I(C)_{n} \rightarrow S_{n} \rightarrow H^{0}\left(C, O_{C}(n)\right) \rightarrow M_{n}(C) \rightarrow 0 .
$$

Let $R=S / I(C)$, so $M(C) \cong H_{*}^{0}\left(C, O_{C}(n)\right) / R$ (where $H_{*}^{0}$ represents the direct sum over all $n$ ).

Now given an element $A+B \cdot X_{2} / G \in H^{0}\left(C, O_{C}(n)\right)$ as in Proposition 3.1, we see that $A$ becomes zero in $M(C)$, and it is a simple exercise to check that $B$ also becomes zero whenever it is a linear combination $P F+Q G$. Also, multiplication by $X_{2}$ and $X_{3}$ are zero in $M(C)$.

We can now prove our main result. Note that the cases $d=0$ and $d=1$ were taken care of in Remark 1.5.

THEOREM 3.4. Let $C$ and $C^{\prime}$ be double lines and assume $p_{a}(C) \leq-2$. Then $C$ is linked to $C^{\prime}$ if and only if

(a) they have the same line $\lambda$ as support,

(b) they have the same arithmetic genus $-d$,

(c) the corresponding maps $\psi, \psi^{\prime}: \lambda \rightarrow \mathbf{P}^{3} / \lambda$ differ by an automorphism of the target $\mathbf{P}^{3} / \lambda$.

Proof. Again, we assume without loss of generality that $C$ is supported on the line $\lambda$ : $\left(X_{2}=X_{3}=0\right)$. Now, assume $C$ is linked to $C^{\prime}$. By Remark 2.3 we may 
assume that $C$ is evenly linked to $C^{\prime}$, so $M(C) \cong M\left(C^{\prime}\right)$ by Hartshorne's theorem (cf. [R1]) and Lemma 1.2. (The latter assures that there is no shift.)

Consider the homomorphism $\phi_{1-d, 1}: S_{1} \rightarrow \operatorname{Hom}\left(M_{1-d}(C), M_{2-d}(C)\right)$. By Corollary 3.3, $\operatorname{ker} \phi_{1-d, 1}=\left\langle X_{2}, X_{3}\right\rangle$, so we have (a). We know (b) from Corollary 1.3. Given these, (c) says that $\left[{ }_{G}^{F}\right]=A\left[F_{G}^{F^{\prime}}\right]$ for some invertible $2 \times 2$ matrix $A$. Equivalently, $F$ and $G$ span the same subspace of $k\left[X_{0}, X_{1}\right]_{d}$ that $F^{\prime}$ and $G^{\prime}$ do.

Consider the homomorphism $\phi_{1-d, d}: S_{d} \rightarrow \operatorname{Hom}\left(M_{1-d}(C), M_{1}(C)\right)$ and let $K$ be its kernel. Let $I(\lambda)$ be the ideal $\left(X_{2}, X_{3}\right) \subset S$. From Corollary 3.3 we have that $K$ is generated by $I(\lambda)_{d}, F\left(X_{0}, X_{1}\right)$ and $G\left(X_{0}, X_{1}\right)$. We thus have

$$
I(\lambda)_{d} \subset K \hookrightarrow S_{d} \stackrel{\phi_{1-d, d}}{\rightarrow} \operatorname{Hom}\left(M_{1-d}(C), M_{1}(C)\right) .
$$

Modding out by $I(\lambda)_{d}$ we then have

$$
\left\langle F\left(X_{0}, X_{1}\right), G\left(X_{0}, X_{1}\right)\right\rangle=K / I(\lambda)_{d} \hookrightarrow S_{d} / I(\lambda)_{d} \cong k\left[X_{0}, X_{1}\right]_{d} .
$$

Since both $K$ and $I(\lambda)$ are isomorphism invariants, we get that the subspace $\langle F, G\rangle$ of $k\left[X_{0}, X_{1}\right]_{d}$ is also, and so we are done.

Conversely, it suffices to check the following: Let $A=\left[\begin{array}{ll}a & b \\ c & d\end{array}\right] \in \mathrm{GL}(2)$. Then

(1) if $b=c=0$, certainly $a \neq 0$ and $d \neq 0$ and we have

$$
A=a\left[\begin{array}{lr}
1 & 0 \\
\frac{d}{a}+1 & -1
\end{array}\right]\left[\begin{array}{ll}
1 & -1 \\
\frac{d}{a}+1 & -1
\end{array}\right]\left[\begin{array}{ll}
1 & -1 \\
0 & -1
\end{array}\right],
$$

(2) if at least one of $b, c$ is nonzero then there exist scalars $\alpha \neq 0, \tau, \mu, \tau^{\prime}, \mu^{\prime}$ such that

$$
A=\alpha\left[\begin{array}{cc}
1 & \mu \\
-\tau & -1
\end{array}\right]\left[\begin{array}{cc}
1 & \mu^{\prime} \\
-\tau^{\prime} & -1
\end{array}\right] .
$$

(The latter is tedious but straightforward.) Then Proposition 2.1 gives the result.

4. Projective geometry and self-linkage. In this section we give an interesting geometric interpretation of the preceding sections which was pointed out by A. Landman. It wil then be easy to say when a double line is self-linked, generalizing a result of $[\mathbf{R 2}]$. For this section, we assume char $k \neq 2$.

As always, we assume that the double line $C$ is supported on the line $\lambda:\left(X_{2}=\right.$ $X_{3}=0$ ), and that $d>1$. The quadric hypersurfaces containing $C$ are then given by homogeneous polynomials of the form $a X_{2}^{2}+b X_{2} X_{3}+c X_{3}^{2}$ (which are always reducible). These hypersurfaces thus form a projective space $\mathbf{P}^{2}$. The ideal generated by two such polynomials is then represented by a line in this $\mathbf{P}^{2}$, or by a point in the dual space $\left(\mathbf{P}^{2}\right)^{*}$. (Henceforth, unless stated otherwise, an ideal shall be understood to be of this special form.)

For the purposes of liaison we need a complete intersection ideal, that is one whose two generators have no common factor. We shall call these allowable ideals. We may thus ask which lines in $\mathbf{P}^{2}$ (or which points in $\left.\left(\mathbf{P}^{2}\right)^{*}\right)$ correspond to allowable ldeals. We now find the complement of this set:

LEMMA 4.1. (a) The "unallowable" ideals form a smooth conic in $\left(\mathbf{P}^{2}\right)^{*}$.

(b) This conic is dual to the "conic of squares" in $\mathbf{P}^{2}$. 


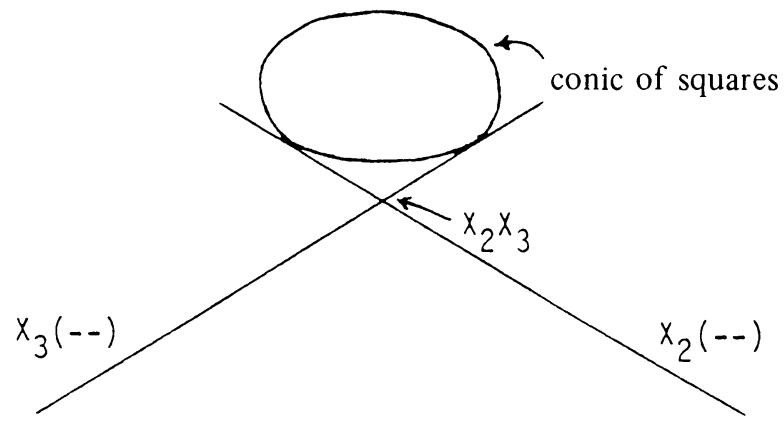

FIGURE 1

Proof. (a) Let $I=\left(a X_{2}^{2}+b X_{2} X_{3}+c X_{3}^{2}, d X_{2}^{2}+e X_{2} X_{3}+f X_{3}^{2}\right)$. One shows using the resultant that these polynomials have a common root if and only if

$$
(a f-c d)^{2}-(a e-b d)(b f-c e)=0 .
$$

Now, the line in $\mathbf{P}^{2}$ through the points $[a, b, c]$ and $[d, e, f]$ has equation

$$
\operatorname{det}\left[\begin{array}{ccc}
Y_{0} & Y_{1} & Y_{2} \\
a & b & c \\
d & e & f
\end{array}\right]
$$

(where $Y_{0}, Y_{1}, Y_{2}$ are the coordinates for this $\mathbf{P}^{2}$ ). Hence the corresponding point in $\left(\mathbf{P}^{2}\right)^{*}$ is $[a e-b d,-(a f-c d), b f-c e]$, and the result follows from (6).

(b) A line in $\left(\mathbf{P}^{2}\right)^{*}$ corresponds to a pencil of lines in $\mathbf{P}^{2}$, i.e. the collection of those ideals containing a given polynomial $L_{1} L_{2}$. This line in $\left(\mathbf{P}^{2}\right)^{*}$ will meet the "unallowable" conic twice in general, corresponding to the ideals $\left(L_{1} L_{2}, L_{1} L_{3}\right)$ and $\left(L_{1} L_{2}, L_{2} L_{3}\right)\left(L_{3}\right.$ arbitrary). This line will be tangent to the "unallowable" conic precisely when the corresponding point in $\mathbf{P}^{2}$ represents a square $L_{1}^{2}$, and so we are done. (Note that the squares really do form a conic in $\mathbf{P}^{2}$, namely $\left.Y_{1}^{2}-4 Y_{0} Y_{2}=0.\right)$

REMARK 4.2. We get several amusing facts about these ideals for free from this description. For example,

(a) A general ideal $\left(L_{1} L_{2}, L_{3} L_{4}\right)$ contains exactly two squares (up to scalar multiplication).

(b) Two distinct ideals of this form have exactly one polynomial in common (up to scalar multiplication).

(c) Given a general polynomial $L_{1} L_{2}$, there are exactly two ideals containing it which are not allowable.

We now consider our basic links of $\S 2$. The set of all polynomials of the form $X_{2}\left(X_{2}+\tau X_{3}\right)$ corresponds to a line in $\mathbf{P}^{2}$, as does the set of all polynomials of the form $X_{3}\left(\mu X_{2}+X_{3}\right)$. These lines meet at $X_{2} X_{3}$ (which is at infinity) and each is tangent to the conic of squares (by duality, since each represents an unallowable ideal). See Figure 1. 
The following is then immediate:

Proposition 4.3. Given an allowable ideal $\left(L_{1} L_{2}, L_{3} L_{4}\right)$, we can find generators which give a basic link if and only if the ideal does not contain the polynomial $X_{2} X_{3}$.

Proof. An allowable ideal corresponds to a line is $\mathbf{P}^{2}$ not tangent to the conic of squares. It then must meet the two distinguished lines either in two distinct points or at the point corresponding to $X_{2} X_{3}$ (see Figure 1).

We now turn to the question of when a double line in self-linked, i.e. when it can be linked to itself in one step. In the case $d=0, C$ is clearly self-linked since it is a plane curve (and so a complete intersection). For $d=1$, on the other hand, it is shown in [R2] that $C$ is self-linked if and only if char $k=2$. This was generalized in [GMV1], where it is shown that if $C_{n}$ is the curve defined by the ideal $\left(I(\lambda)^{n}, X_{0} X_{3}-X_{1} X_{2}\right)$, then $C$ is never self-linked for $n>2$. ( $n=1$ is Rao's result.) We now give a different generalization:

THEOREM 4.4. A double line $C$ of arithmetic genus $<-1$ (i.e. $d \geq 2$ ) is self-linked if and only if char $k=2$.

PROOF. In view of Remark 2.4, the only thing left to prove is that for char $k \neq 2$, $C$ cannot be self-linked by a nonbasic link.

By Proposition 4.3, "almost all" links are basic. Hence we have only to consider allowable ideals of the form $\left(X_{2} X_{3}, L_{1} L_{2}\right)$. Clearly we may write this as $\left(X_{2} X_{3}, a X_{2}^{2}+b X_{3}^{2}\right)$, where $a \neq 0$ and $b \neq 0$. Then writing $I(C)$ and the linked ideal $I\left(C^{\prime}\right)$ as in $\S 2$, a similar calculation shows

$$
\left[\begin{array}{l}
F^{\prime} \\
G^{\prime}
\end{array}\right]=\alpha\left[\begin{array}{ll}
0 & a \\
b & 0
\end{array}\right]\left[\begin{array}{l}
F \\
G
\end{array}\right] .
$$

Therefore $C$ is not self-linked.

REMARK 4.5. We can now generalize Proposition 2.1 to say that $C$ is directly linked to $C^{\prime}$ if and only if

where $\operatorname{det} A \neq 0$ and trace $A=0$.

$$
\left[\begin{array}{l}
F^{\prime} \\
G^{\prime}
\end{array}\right]=A\left[\begin{array}{l}
F \\
G
\end{array}\right]
$$

\section{REFERENCES}

[GMV1] A. Geramita, P. Maroscia and W. Vogel, On curves linked to lines in $\mathbf{P}^{3}$, The Curves Seminar at Queen's, II, Queen's Papers in Pure and Applied Math., vol. 61, Kingston, Ontario, 1982, pp. B1-B26.

[GMV2] _ A note on arithmetically Buchsbaum curves in $\mathbf{P}^{3}$, Queen's University Preprint No. 1983-24.

[H] J. Harris, Curves in projective space, Les Presses de L'Université de Montréal, 1982.

[Ha] R. Hartshorne, Algebraic geometry, Springer-Verlag, New York, 1977.

[M] J. Migliore, Geometric invariants for liaison of space curves, J. Algebra (to appear).

[R1] P. Rao, Liaison among curves in $\mathbf{P}^{3}$, Invent. Math. 50 (1979), 205-217.

[R2] _ _ On self-linked curves, Duke Math. J. 49 (1982), 251-273.

Department of MATHEMATICS, NORThWESTERn UNIVERSity, EVANSTON, ILlinois 60201

Current address: Department of Mathematics, Drew University, Madison, New Jersey 07940 